\title{
Identification and functional characterization of the CYP51 gene from the yeast Xanthophyllomyces dendrorhous that is involved in ergosterol biosynthesis
}

\author{
Kritsye Leiva, Nicole Werner, Dionisia Sepúlveda, Salvador Barahona, Marcelo Baeza, Víctor Cifuentes \\ and Jennifer Alcaíno*
}

\begin{abstract}
Background: Xanthophyllomyces dendrorhous is a basidiomycetous yeast that synthesizes astaxanthin, a carotenoid with great biotechnological impact. The ergosterol and carotenoid synthetic pathways derive from the mevalonate pathway and involve cytochrome P450 enzymes. Among these enzymes, the CYP51 family, which is involved in ergosterol biosynthesis, is one of the most remarkable that has C14-demethylase activity.

Results: In this study, the CYP51 gene from X. dendrorhous was isolated and its function was analyzed. The gene is composed of ten exons and encodes a predicted 550 amino acid polypeptide that exhibits conserved cytochrome P450 structural characteristics and shares significant identity with the sterol C14-demethylase from other fungi. The functionality of this gene was confirmed by heterologous complementation in S. cerevisiae. Furthermore, a CYP51 gene mutation in $X$. dendrorhous reduced sterol production by approximately $40 \%$ and enhanced total carotenoid production by approximately $90 \%$ compared to the wild-type strain after 48 and $120 \mathrm{~h}$ of culture, respectively. Additionally, the CYP51 gene mutation in X. dendrorhous increased HMGR (hydroxy-methylglutaryl-CoA reductase, involved in the mevalonate pathway) and crtR (cytochrome P450 reductase) transcript levels, which could be associated with reduced ergosterol production.
\end{abstract}

Conclusions: These results suggest that the CYP51 gene identified in X. dendrorhous encodes a functional sterol C14-demethylase that is involved in ergosterol biosynthesis.

Keywords: Cytochrome P450 enzyme systems (P450), Astaxanthin, Sterols, Sterol C14-demethylase

\section{Background}

Xanthophyllomyces dendrorhous is a basidiomycete yeast that has been mostly studied for its ability to synthesize xanthophyll astaxanthin as its main carotenoid. Astaxanthin (3,3'-dihydroxy- $\beta$, $\beta$-carotene-4-4'-dione) takes third place in the global market for carotenoids, which reached $\$ 226$ million in the year 2010 and is expected to exceed $\$ 250$ million by the year 2018 [1]. This carotenoid is currently used in aquaculture for salmon flesh pigmentation and as a supplement for the diets of farm chickens to

\footnotetext{
*Correspondence: jalcainog@u.uchile.cl
Departamento de Ciencias Ecológicas y Centro de Biotecnología, Facultad de

*Correspondence: jalcainog@u.uchile.cl Ciencias, Universidad de Chile, Las Palmeras 3425, Casilla 653, Ñuñoa, Santiago, Chile
}

produce a stronger yolk and flesh pigmentation [2]. Additionally, astaxanthin has strong antioxidant properties and has been proposed to play a protective role against oxidative stress in yeast [3-5]. Recent findings suggested that astaxanthin supplementation could be beneficial as a treatment for several degenerative diseases in addition to other potential benefits for human health [6-9]. To date, $X$. dendrorhous is the only known organism that synthesizes astaxanthin from $\beta$-carotene through a cytochrome entem [10-13], suggesting that this yeas production of this carotenoid.

Cytochrome P450 proteins (P450s or CYPs) represent a large superfamily of heme-containing monooxygenases 
that are distributed throughout three domains of life [14] and play significant roles in the oxidative metabolism of a wide range of exogenous and endogenous substrates [15]. CYP enzymes are involved in the biosynthesis of many physiologically important compounds, such as sterols, steroid hormones, fatty acids and vitamins [16], and a vast array of secondary metabolites in plants, insects and fungi [17]. Additionally, these enzymes are the main catalysts involved in the activation and detoxification of different xenobiotics, such as drugs, pesticides, carcinogens and environmentally contaminating chemicals [18]. CYP enzymes require the reducing equivalents from NADPH or $\mathrm{NADH}$, which are generally transferred to CYPs through a redox partner to catalyze the general reaction $\mathrm{RH}+\mathrm{O}_{2}+2 \mathrm{e}^{-}+2 \mathrm{H}^{+} \rightarrow \mathrm{ROH}+\mathrm{H}_{2} \mathrm{O}[19]$.

Only two CYP enzymes have been described in $X$. dendrorhous: the astaxanthin synthase that is involved in astaxanthin biosynthesis and CYP61, which participates in ergosterol biosynthesis [20]. Considering the biological relevance of this protein family and the involvement of a unique system in $X$. dendrorhous, in this work we describe a third CYP in this yeast: CYP51. CYP51 is one of the first modifying enzymes involved in sterol synthesis. It catalyzes the C14 demethylation of lanosterol in yeasts during ergosterol biosynthesis and is considered to be an ancestral CYP from which other CYPs evolved [21].

\section{Results and discussion}

\section{Cloning and sequence analysis of the $X$. dendrorhous CYP51 gene}

Using bioinformatic analyses of the genomic and transcriptomic data of $X$. dendrorhous available in our laboratory with the $S$. cerevisiae ERG11 gene as a query [GenBank: NM_001179137], we identified a putative $X$. dendrorhous CYP51 gene. This gene was uploaded into the Genbank database [GenBank: KP317478]. The primers CYP51 up.F and CYP51dw.R were designed based on this gene sequence and used to amplify genomic DNA from strain UCD 67-385. The obtained PCR-product of approximately 4,300 bp was inserted into the EcoRV site of the pBluescript SK- plasmid, generating plasmid pBS-gCYP51. Similarly, the cDNA version (ORF: 1,653 bp) of the CYP51 gene was obtained by RT-PCR from $X$. dendrorhous total RNA using the primer set cCYP51.F + cCYP51.R. This cDNA was ligated into the EcoRV site of the pBluescript SK- plasmid to generate plasmid pBS-cCYP51. The sequences of the genomic and cDNA versions were determined in both strands and compared, revealing that the CYP51 gene from $X$. dendrorhous consists of 10 exons of 141, 70, 47, 63, 60, 462, 86, 233, 83 and 408 bp and 9 introns of 356, 95, 79, 80, 86, 110, 90, 87 and 90 bp. The CYP51 gene was predicted to encode a 550 amino acid protein with an expected molecular weight of $61.8 \mathrm{kDa}$ and $\mathrm{pI}$ of 6.64 . This protein shares $48 \%$ identity and $65 \%$ similarity at $91 \%$ coverage with the $S$. cerevisiae C14-demethylase [ERG11, Swiss-Prot: P10614] (Figure 1), which belongs to the cytochrome $\mathrm{P} 450$ protein family and is involved in the third step of ergosterol biosynthesis from squalene: the conversion of lanosterol to 4,4-dimethylcholesta8,14,24-trienol [22,23].

Amino acid sequences are highly diverse among the cytochrome P450 protein family; however, their structural fold is highly conserved [24]. In the deduced CYP51 protein from $X$. dendrorhous, several cytochrome P450 secondary structural elements were predicted using the CYP450 Engineering database, including alpha helices A, B, C, E, F, G, H, I, J, K, K' and L, beta-sheets 1-1,1-2, $1-5,3-1,1-4,2-1,2-2,1-3,3-3,4-1,4-2$ and $3-2$, the meander loop involved in the stabilization of the tertiary structure and heme binding, and the Cys pocket that contains the conserved cysteine involved in heme binding [25] (Figure 1). Identification of P450s across biological kingdoms depends largely on the identification of two P450 signature motifs: ExxR at the K-helix and FxxGxRxCxG (also known as the CxG motif) at the Cys-pocket. These motifs contain three totally conserved amino acids: the glutamic acid and arginine of the ExxR motif and the cysteine in the Cys pocket that serves as a fifth ligand for the heme iron [26]. Site-directed mutagenesis of these three invariant residues in most CYPs results in inactive and misfolded P450 isoforms, suggesting their importance in maintaining the P450 structure [27,28]. All of these motifs were recognized in the $X$. dendrorhous deduced CYP51 protein, including the three invariant P450 residues. A threonine and a leucine were found at the second and third positions of the ExxR motif; these are the preferred residues at these positions in P450s, including the CYP51 family [29]. Moreover, the CxG motif of the $X$. dendrorhous CYP51 protein (FGAGRHRCIG) maintains the preferred residues described for the CYP51 family [29]. This finding is interesting because instead of the conserved arginine at the sixth position of this motif, CYP51 contains a histidine surrounded by arginines. Additionally, a putative hydrophobic trans-membrane segment at the CYP51 amino terminus was predicted. This finding represents an important feature that allows class II P450 enzymes to anchor to the endoplasmic reticulum [30]. These observations strongly suggest that the identified $X$. dendrorhous gene encodes a cytochrome P450 enzyme, specifically CYP51 with lanosterol demethylase activity.

The deduced $X$. dendrorhous CYP51 protein was modeled (Figure 2A) with the homology modeling technique using the SwissModel web server. The $S$. cerevisiae ERG11 protein [PDB: 4 lxj.1], which shares $48 \%$ sequence identity, was used as a template. Additionally, possible protein-ligand binding interactions between the 


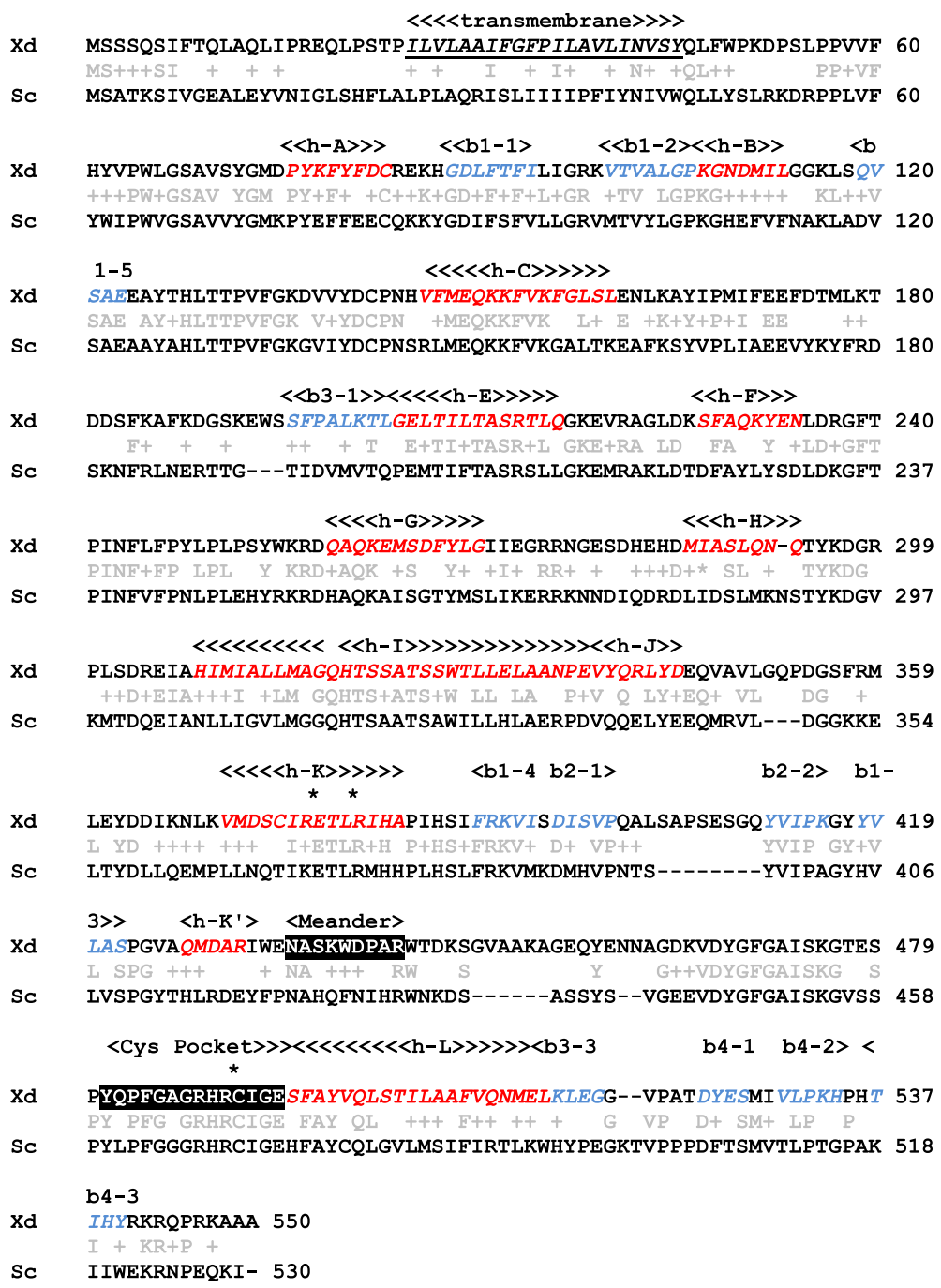

Figure 1 Sequence alignment between $X$. dendrorhous CYP51 and S. cerevisiae ERG11 proteins and prediction of structurally conserved motifs in CYP51. Amino acid alignment between the deduced CYP51 sequence from X. dendrorhous (Xd) strain UCD 67-385 and the S. cerevisiae (Sc) strain S288C ERG11 (lanosterol C14-demethylase) protein [Swiss Protein: P10614]. Amino acid differences with the same properties are denoted with a plus (+). Structural elements are highlighted with the name of the corresponding feature above them: possible transmembrane helix (underlined and italics), alpha helices (red and italics), beta-sheets (blue and italics), meander loop and Cys pocket (white highlighted in black). The asterisks $\left(^{*}\right)$ indicate the three totally conserved amino acids among cytochromes P450. Secondary structural elements were predicted with the CYP450 Engineering database [26], and the potential transmembrane region was predicted with TMpred [52].

CYP51 protein model and the potential lanosterol and itraconazole ligands were predicted with the protein docking technique using the AutoDock4 program [31] (Figure 2B). According to the results, the azole antifungal fits in a similar way to the substrate lanosterol in a hydrophobic groove and interacts with Met-315 and the P-methyl group of Thr-322. Previous studies have also reported these residues as binding sites of azole antifungals in cytochrome P450 proteins [32]. Therefore, the itraconazole azole binds to the CYP51 protein in the substrate-binding pocket of the enzyme, thus blocking its enzymatic catalysis.

\section{Functional analysis of the $X$. dendrorhous CYP51 gene}

The functionality of the $X$. dendrorhous CYP51 gene was evaluated by two different approaches: heterologous complementation in $S$. cerevisiae and gene mutation in $X$. dendrorhous.

The heterologous complementation analyses were performed using a diploid $S$. cerevisiae that is heterologous for the ERG11 gene (Sc-erg11 ${ }^{+/}$strain), because a null mutant is not viable [33]. The $S$. cerevisiae ERG11 gene and the cDNA of the $X$. dendrorhous CYP51 gene were ligated into the $S$. cerevisiae expression vector YEpNP, generating plasmids YEpNP-gERG11 and YEpNP-cCYP51; 


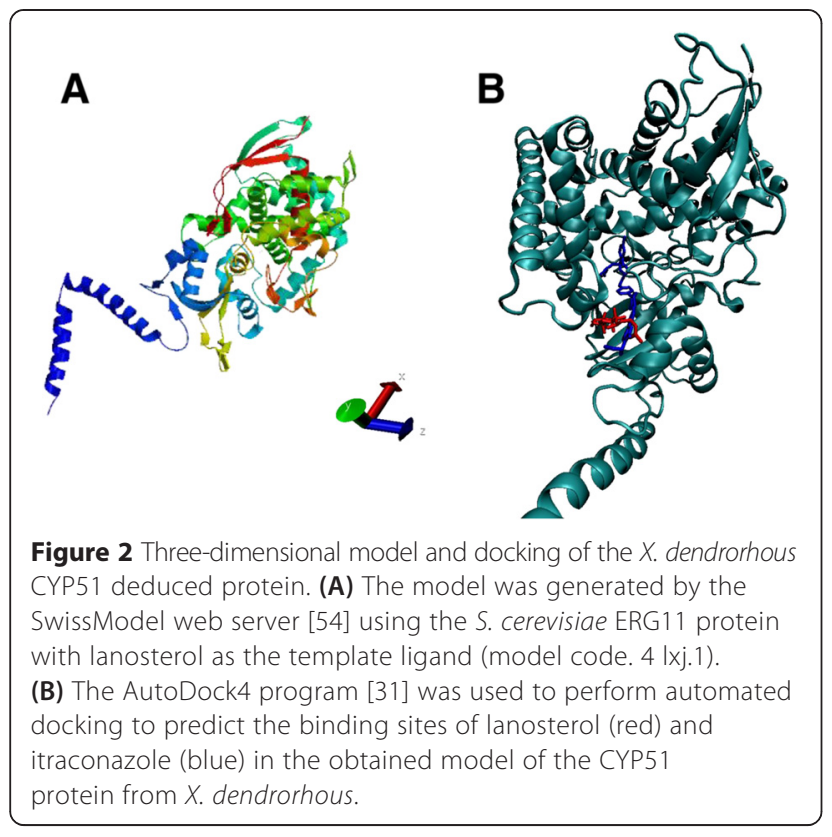

then, these plasmids were used to transform the Sc-erg $11^{+/-}$ strain. Several transformants were recovered in each case, and at least three transformants of each type were randomly chosen to confirm that they contained the respective plasmid. Given that all of the analyzed transformants showed a positive result in this analysis, one of each (named Sc-gERG11Sc and Sc-cCYP51Xd, respectively) was chosen for sporulation to obtain haploid strains. After 6 days of incubation in sporulation media agar plates, the formation of asci was confirmed by optical microscopy. The asci were broken and seeded onto SD agar plates supplemented with G418 to confirm the presence of the S. cerevisiae ERG11 mutant allele, and with uracil, histidine, lysine and methionine to sustain all possible auxotrophies in the resulting haploid strains. Although the haploid cells were enriched by diethyl ether treatment [34], it is also possible to obtain diploid strains among the G418-resistant strains. Because of this, haploid strains were first selected according to their auxotrophy for methionine and lysine, both of which are heterozygous markers in the original diploid parental strain. Subsequently, the haploid condition of the ERG11 mutant allele and its complementation of the expression vector were confirmed by PCR analyses using comprehensive sets of primers (Figure 3). After these analyses, a single strain that fulfilled the requirements for heterologous expression studies carrying the YEpNP-gERG11 or the YEpNP-cCYP51 vector was chosen; these strains were named Sc-hERG11 and Sc-hCYP51, respectively.

The strains Sc-hERG11 and Sc-hCYP51 did not show significant differences in their growth curves when cultured in $\mathrm{YM}$ medium at $22^{\circ} \mathrm{C}$ with constant agitation
(Additional file 1: Figure S1). Additionally, sterols were extracted after 10, 24 and $56 \mathrm{~h}$ of cultivation (Table 1). When the sterol compositions were analyzed by RPHPLC, all strains showed the same sterol pattern, with one main sterol produced with $13 \mathrm{~min}$ of retention time. This sterol was confirmed to correspond to ergosterol after co-injecting each sample with standard ergosterol. In general, strain Sc-hCYP51 showed a lower sterol content than strains Sc-erg11 $1^{+-}$and Sc-hERG11 at all analyzed time points. However, the fact that strain Sc-hCYP51 was viable and able to produce ergosterol indicates that the CYP51 gene from $X$. dendrorhous complements the erg11 null mutation in S. cerevisiae. Therefore, this gene encodes a lanosterol C14-demethylase that is able to couple with the endogenous S. cerevisiae cytochrome $\mathrm{P} 450$ reductase in vivo.

To evaluate the functionality of CYP51 in X. dendrorhous, cyp51 mutants were created by replacing the CYP51 locus with an antibiotic resistance module through homologous recombination as previously described [35]. For this purpose, the wild-type strain CBS 6938 was chosen because it has been shown to be aneuploid, and hemizygous mutants can be obtained after only one transformation event [12]. Before transformation, the CYP51 gene from strain CBS 6938 was sequenced, uploaded to the Genbank database [Genbank: KP317479] and compared to the sequence from strain UCD 67-385. Minimal differences were found between these two strains, which possessed $99.707 \%$ identity in the region from the start to the stop translation codons. After CBS 6938 transformation with linear plasmid pBScyp51/hph, a hygromycin B-resistant transformant was obtained. Although it was confirmed that the CYP51 locus was indeed replaced by the resistance module in this strain, the CYP51 gene could still be amplified using a comprehensive set of primers, suggesting that this strain was heterozygous for the CYP51 locus. Therefore, this strain was named CBS-CYP51 $1^{+-}$. This strain was still a useful model to evaluate the effect of the CYP51 mutation in $X$. dendrorhous; because the deletion of this gene has been shown to be lethal to the cells of other yeast strains, a cyp51 null mutant would most likely not be viable [36]. Interestingly, the mutant strain had a more intense reddish color that was discernable to the naked eye than the wild type strain, suggesting that it produces more carotenoids than the parental strain. To confirm this observation and to evaluate phenotypic variations between these two strains, growth curves were constructed and sterols and carotenoids were extracted and quantified after 48 and $120 \mathrm{~h}$ of cultivation.

The growth curves of both strains did not show significant differences at the lag and exponential phases of growth; however, strain CBS-CYP51 ${ }^{+/-}$reached a lower $\mathrm{OD}_{600}$ during the stationary phase of growth (P-value < 0.05 , Student's $t$ test) (Additional file 1: Figure S1). After 

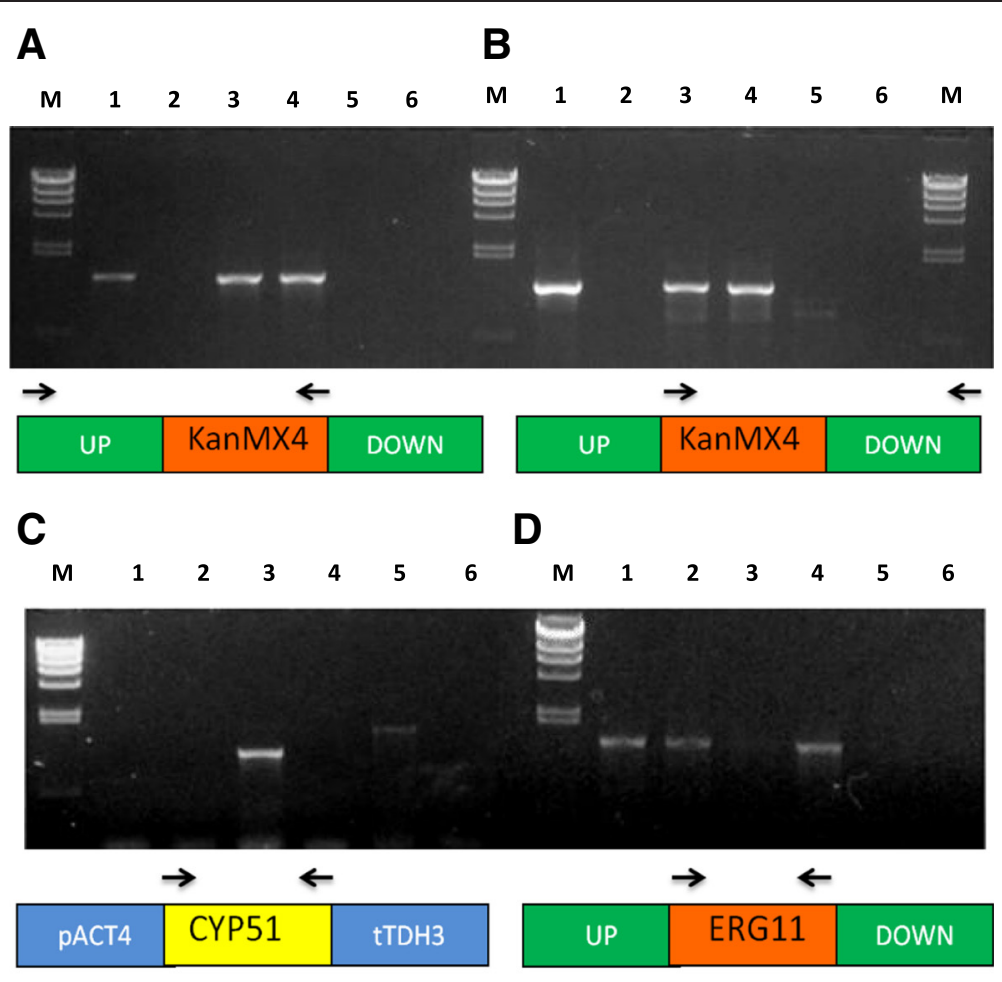

\section{E}

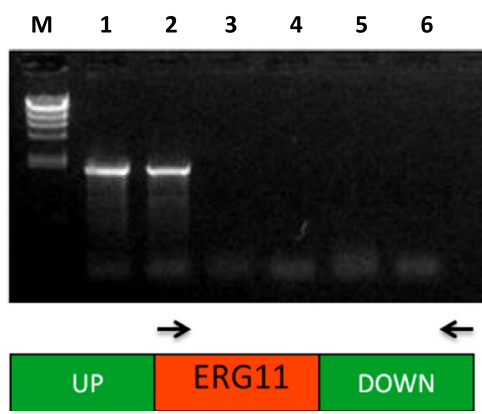

Figure 3 PCR-based analyses of S. cerevisiae erg $11^{-}$strains carrying the YEpNP-gERG11 and YEpNP-cCYP51 vectors. PCR analyses to confirm the presence of the kanMX gene at the ERG11 locus (panel A: primers CYP51ScEXt.F and KanMX4.R2 and panel B: primers KanMX4.F2 and CYP51ScExt.R), the presence of the X. dendrorhous CYP51 gene (panel C: primers RTCYP51.F and CYP51.Rb), the presence of the ERG11 gene (panel D: gERG11.F and gERG11.R) and the absence of the ERG11 gene in the S. cerevisiae genome (panel E: gERG11.F and CYP51SCExt.R). Template DNA in each lane: S. cerevisiae parental diploid strain erg11 +/- (Lane 1), S. cerevisiae S288c strain (Lane 2), S. cerevisiae Sc-hCYP51 strain (Lane 3), S. cerevisiae Sc-hERG11 strain (Lane 4), X. dendrorhous UCD 67-385 strain (Lane 5), negative control without DNA (Lane 6). Molecular size marker Lambda/Hind III (Lane M: 23.1, 9.4, 6.6, 4.4, 2.3, 2.0 and $0.6 \mathrm{~kb}$ ). A schematic diagram is included to represent the primer sets (shown in arrows) that were used. The UP and DOWN regions correspond to regions located 300 bp upstream and downstream of the S. cerevisiae ERG11 gene, respectively. Region KanMX4 corresponds to the geneticin (G418) resistance module and the PACT4 and tTDH3 regions correspond to the S. cerevisiae promoter and terminator region in YEpNP.

$48 \mathrm{~h}$ of cultivation, the wild-type strain accumulated approximately $70 \%$ more sterols than the mutant strain; however, after $120 \mathrm{~h}$ of culture, there were no statistically significant differences between the strains (Table 2). This outcome may be the result of a regulation mechanism that modulates sterol biosynthesis according to the metabolic state of the yeast, which is reduced during the stationary phase of growth. Similarly, previous studies in filamentous fungi and in yeast have reported reduced ergosterol accumulation during the stationary phase of growth [20,37]. Moreover, both strains showed the same sterol composition, with ergosterol representing close to $100 \%$ of the identified sterols; this result was confirmed by co-injection of the samples with standard ergosterol in RP-HPLC analysis. An additional two unidentified sterols could be detected in both strains with retention 
Table 1 Sterols obtained from S. cerevisiae strains used in this study (mg per $\mathbf{g}$ of dry yeast)

\begin{tabular}{|c|c|c|c|c|c|c|c|c|c|}
\hline \multirow[b]{3}{*}{ Cultivation Time (h) } & \multicolumn{9}{|c|}{ S. cerevisiae strains } \\
\hline & \multicolumn{3}{|c|}{$\left(\right.$ Sc-erg $\left.11^{+/-}\right)$} & \multicolumn{3}{|c|}{ Sc-hERG11 } & \multicolumn{3}{|c|}{ Sc-hCYP51 } \\
\hline & 10 & 24 & 56 & 10 & 24 & 56 & 10 & 24 & 56 \\
\hline Total sterols & $25 \pm 5$ & $11 \pm 1$ & $21 \pm 3$ & $21 \pm 8$ & $16 \pm 5$ & $38 \pm 7$ & $19 \pm 3$ & $9 \pm 1$ & $20 \pm 2$ \\
\hline Ergosterol (11 min) & $23 \pm 5$ & $10 \pm 1$ & $20 \pm 2$ & $17 \pm 2$ & $15 \pm 4$ & $34 \pm 6$ & $16 \pm 3$ & $9 \pm 3$ & $18 \pm 2$ \\
\hline Peak 2 (12.5 min) & ND & $0.03 \pm 0.02$ & $0.2 \pm 0.3$ & ND & $0.06 \pm 0.02$ & $0.4 \pm 0.4$ & ND & ND & $0.21 \pm 0.04$ \\
\hline Peak 3 (15 min) & $3 \pm 1$ & $0.7 \pm 0.1$ & $1.45 \pm 0.03$ & $4 \pm 1$ & $1.5 \pm 0.4$ & $3.1 \pm 0.4$ & $2.4 \pm 0.6$ & $0.7 \pm 0.1$ & $1.6 \pm 0.1$ \\
\hline
\end{tabular}

ND: Not detected. Table values correspond to the average result from three independent cultures \pm standard deviations. The RP-HPLC retention time is indicated in parenthesis.

times of 12.5 and $15 \mathrm{~min}$; however, these sterols were found in a significantly lower proportion.

The mutant strain contained a higher amount of carotenoids (P-value $<0.05$, Student's $t$ test), with an approximately 1.9 -fold increase after $120 \mathrm{~h}$ of culture. Previous studies on carotenogenesis in $X$. dendrorhous have shown that this process is induced by the end of the exponential and early stationary phases of growth, coinciding with the point at which glucose is completely exhausted [38-40]. Therefore, it is expected that the major differences in carotenoid content can be better appreciated in older cultures. However, the CBS-CYP51 $1^{+/-}$strain also had a higher carotenoid content after $48 \mathrm{~h}$ of culture, suggesting that carotenogenesis starts earlier in this strain. Moreover, carotenoid composition also differed between both strains, with a reduced astaxanthin proportion in the CBS-C $\mathrm{P} 1^{+/-}$strain (Table 2). Interestingly, changes in the carotenoid composition showed similarity to cultures of $X$. dendrorhous in which the crtI gene (encoding the phytoene desaturase) was over-expressed [41], resulting in an increased monocyclic carotenoid proportion in CBS-CYP51 ${ }^{+/-}$cells. Moreover, $\beta$-carotene and xanthophyll intermediaries such as phoenicoxanthin and canthaxathin are increased during the synthesis of astaxanthin from $\beta$-carotene in CBS-CYP51 $1^{+/-}$, suggesting that the cytochrome $\mathrm{P} 450$ system involved in these steps may be weakened. It is likely that this change is the consequence of the reduced CYP51 gene dose in CBS-CYP51 $1^{+/}$, which could favor the activity of the cytochrome P450 systems involved in ergosterol biosynthesis at the expense of astaxanthin synthesis. This idea is supported by the higher cytochrome P450 reductase transcript levels found in the CBS-CYP51 ${ }^{+/}$strain after $24 \mathrm{~h}$ of cultivation (see below) that can sustain cytochrome P450 activity during ergosterol biosynthesis.

Similar to our findings, several studies have observed increased carotenoid production in $X$. dendrorhous when ergosterol levels are reduced. For example, when Phaffia rhodozyma (the anamorphic state of $X$. dendrorhous) was cultured in the presence of the antifungal ergosterol biosynthesis inhibitor fluconazole, enhanced astaxanthin production was observed [42]. One possible explanation is that ergosterol down-regulates its own synthesis via a negative-feedback mechanism. Therefore, a reduced ergosterol content favors the availability of mevalonate pathway products, which are common to ergosterol and carotenoid syntheses. This reasoning is supported by a previous study in which $X$. dendrorhous mutants that are

Table 2 Sterols (mg per $\mathrm{g}$ of dry yeast) and carotenoids ( $\mu \mathrm{g}$ per $\mathrm{g}$ of dry yeast) obtained from the $X$. dendrorhous used in this study

\begin{tabular}{|c|c|c|c|c|}
\hline \multirow[b]{3}{*}{ Cultivation Time (h) } & \multicolumn{4}{|c|}{$X$. dendrorhous strains } \\
\hline & \multicolumn{2}{|l|}{ CBS 6938} & \multicolumn{2}{|l|}{ CBS-CYP51 $1^{+/-}$} \\
\hline & 48 & 120 & 48 & 120 \\
\hline Sterols: & $5.6 \pm 0.2$ & $3.8 \pm 0.4$ & $3.2 \pm 0.7$ & $4.9 \pm 0.8$ \\
\hline Total Carotenoids: & $24 \pm 5(100)$ & $256 \pm 18(100)$ & $325 \pm 115(100)$ & $486 \pm 54(100)$ \\
\hline Astaxanthin & $19 \pm 4(78)$ & $174 \pm 23(68)$ & $95 \pm 27(29)$ & $203 \pm 20(42)$ \\
\hline Phoenicoxanthin & $2.5 \pm 0.7(10)$ & $30.0 \pm 0.8(12)$ & $37 \pm 15(11)$ & $74 \pm 14(15)$ \\
\hline Canthaxanthin & ND & ND & $9 \pm 5(3)$ & $12 \pm 6(3)$ \\
\hline Echinenone & ND & ND & $4 \pm 7(1)$ & ND \\
\hline$\beta$-carotene & ND & $12 \pm 6(5)$ & $67 \pm 33(21)$ & $55 \pm 15(11)$ \\
\hline Lycopene & ND & ND & $7 \pm 6(2)$ & ND \\
\hline Monocyclic carotenoids & $2.8 \pm 0.8(11)$ & $40 \pm 3(16)$ & $107 \pm 40(33)$ & $149 \pm 27(31)$ \\
\hline
\end{tabular}

ND: Not detected. Table values correspond to the average result from three independent cultures \pm standard deviations. Percentage relative to total carotenoids is indicated in parenthesis. Monocyclic carotenoids include: $\gamma$-carotene, keto- $\gamma$-carotene, hydroxy-keto- $\gamma$-carotene, torulene and hydroxy-keto-torulene. 
unable to synthesize ergosterol due to the cyp61 mutation had increased carotenoid content compared to their parental wild type strains. Moreover, transcript levels of at least one gene of the mevalonate pathway (HMGR that encodes the hydroxymethylglutaryl CoA-reductase) were significantly increased [20]. In this sense, the supplementation of mevalonate in P. rhodozyma cultures resulted in an increase in carotenoid production [43]. Furthermore, deletion of the gene encoding squalene synthase (ERG9, the first step of sterol biosynthesis per se) in combination with the overexpression of the catalytic domain of the hydroxymethylglutaryl CoA-reductase in a recombinant Candida utilis strain caused an increase in lycopene production [44].

Based on this reasoning, we evaluated whether HMGR transcript levels were enhanced in strain CBS-CYP51 ${ }^{+/}$. To accomplish this, total RNA was extracted from the parental and CBS-CYP51 ${ }^{+/-}$strains after 24 and $48 \mathrm{~h}$ of cultivation in YM media with constant agitation at $22^{\circ} \mathrm{C}$, and the transcript levels were analyzed by RT-qPCR. The results revealed that the HMGR transcript level was indeed higher in the mutant strain compared to the wild-type strain, indicating that the elimination of one allele of the CYP51 gene of $X$. dendrorhous affected the expression of HMGR (Figure 4A). The HMGR gene controls one of the key regulatory steps in the mevalonate pathway, and, in turn, carotenoid and ergosterol biosynthesis through substrate availability [45]; therefore, an increase in the HMGR transcript level could partly explain the increased carotenoid content in the mutant strain CBS-CYP51 ${ }^{+/-}$. Similar to our findings, there was an increase in carotenoid content that was also related to a higher HMGR transcript levels following the treatment of the fungus Blaskelea trispora with ketoconazole, a specific inhibitor of CYP51 [46].
Because cytochrome P450 enzymes are involved in both astaxanthin and ergosterol biosynthesis, we also evaluated the $c r t R$ transcript levels [12]. $c r t R$ encodes for a cytochrome $\mathrm{P} 450$ reductase, which is the main electron donor in these systems. Interestingly, the $\mathrm{crt} R$ transcript level was also higher in CBS-CYP51 ${ }^{+/-}$after $24 \mathrm{~h}$ of cultivation; however, this difference was not statistically significant after $48 \mathrm{~h}$ of growth (Figure 4B). Although several different genes may encode cytochrome P450 enzymes in an organism, usually there is only one gene encoding the enzyme cytochrome P450 reductase. Consequently, a complex mechanism of regulation of the expression of $c r t R$ is required to set the levels of cytochrome P450 reductase activity in relation to the various cytochrome P450 proteins in this organism. To date, three cytochrome P450-encoding genes have been described in $X$. dendrorhous: crtS [10], CYP61 [20] and CYP51 [this current work]). Thus, the cytochrome $\mathrm{P} 450$ reductase would act as a cytochrome P450 redox partner at least in the carotenoid and ergosterol synthesis pathways. Finally, we also analyzed whether the elimination of one CYP51 allele indeed affected its own transcript levels. After $24 \mathrm{~h}$ of cultivation, no significant differences were observed between the mutant and the parental strain; however, after $48 \mathrm{~h}$ of cultivation, differences could be appreciated (Figure 4C), confirming that the $X$. dendrorhous mutant generated in this work indeed affected CYP51 transcript levels.

\section{Conclusions}

In this work, the CYP51 gene from $X$. dendrorhous was identified and its function was characterized. This gene encodes a putative protein that contains all of the cytochrome P450 conserved motifs and is consistent with CYP51 protein family characteristics. The heterologous complementation analysis in S. cerevisiae strongly suggests

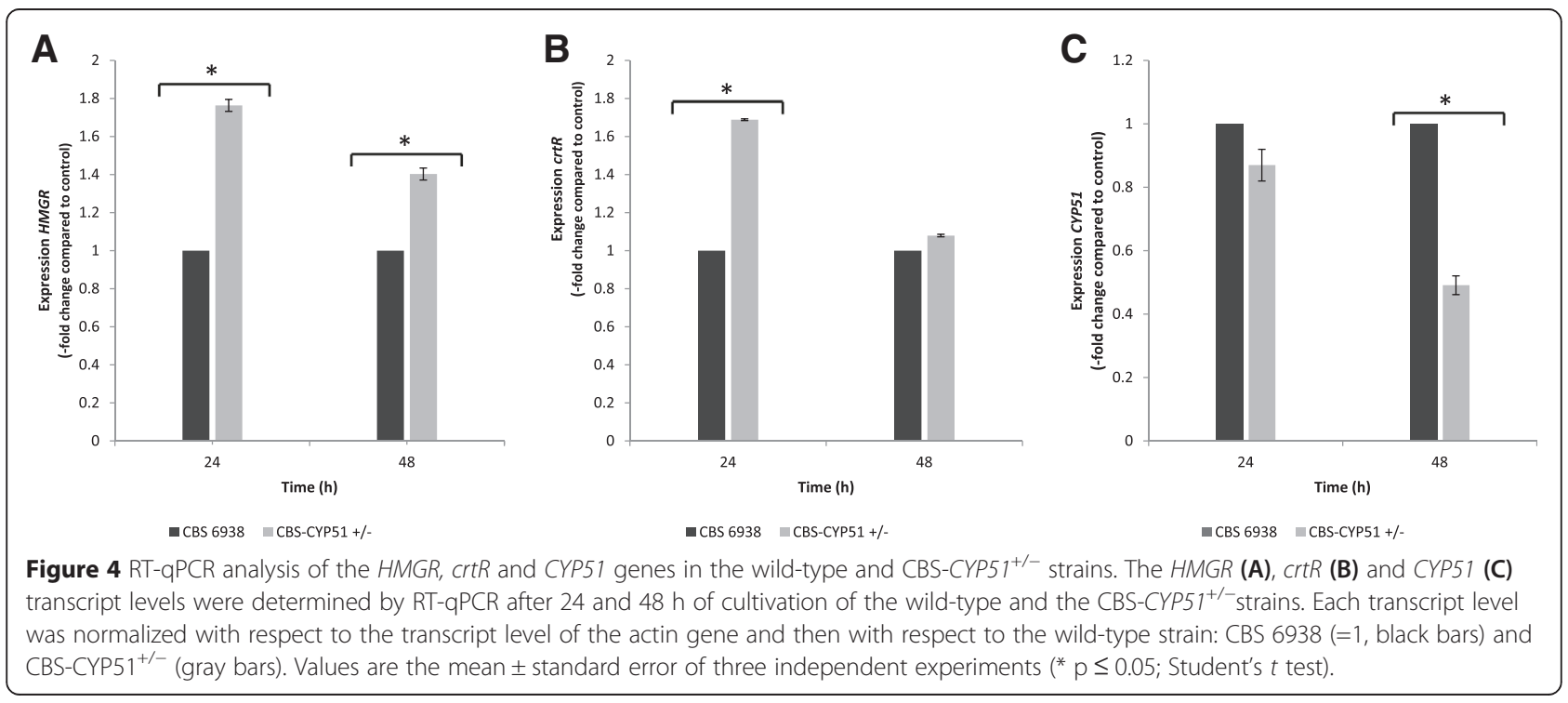


that this gene in fact encodes a functional sterol C14demethylase. Moreover, a CYP51 mutation in X. dendrorhous affected sterol and carotenoid production, supporting the finding that reduced sterol production is related to higher carotenoid production.

\section{Methods}

Biological material and microorganism culture conditions Restriction enzymes, the Klenow polymerase and the MMLV reverse transcriptase were purchased from Promega, and the Pfu DNA polymerase was purchased from Invitrogen. The plasmids and microbial strains that were used and constructed for this work are included in Table 3. The wild type UCD 67-385 (ATCC 24230) X. dendrorhous strain was used for genome and transcriptome sequencing and for genomic and cDNA CYP51 gene amplification and isolation. For the CYP51 gene mutation, the wild type CBS 6938 (ATCC 96594) X. dendrorhous strain was used. Escherichia coli DH-5 $\alpha$ was used as a host for plasmid propagation. Saccharomyces cerevisiae Meyen ex E.C Hansen YHR007C BY4743 (ATCC 26604) was used for CYP51 heterologous complementation analysis because it is a diploid and heterozygous mutant for the ERG11 gene $\left(\operatorname{erg} 11^{(+/)}\right)$, with one copy of ERG11 replaced by a geneticin resistance cassette. Most plasmid constructs derived from pBluescript SK- [47]. Plasmid YEpNP was modified from YEpACT4 [48] and used for S. cerevisiae heterologous complementation.

$X$. dendrorhous was cultured in YM rich medium (1\% glucose, $0.3 \%$ yeast extract, $0.3 \%$ malt extract and $0.5 \%$ peptone) with constant agitation at $22^{\circ} \mathrm{C}$. For transformant selection, the yeast was grown on $1.5 \%$ agar $\mathrm{YM}$

Table 3 Plasmids and strains used and constructed in this study

$$
\text { Relevant features or strain genotype }
$$

Source or

Reference

\section{Plasmids:}

\begin{tabular}{|c|c|c|}
\hline pBluescript SK- (pBS) & ColE1 ori; AmpR; cloning vector with blue-white selection. & Invitrogen \\
\hline pMN-hph & $\begin{array}{l}\text { pBS containing at the EcoRV site a cassette of } 1.8 \mathrm{~kb} \text { bearing the } E \text {. coli-Hygromycin B resistance } \\
(h p h) \text { gene under EF-1 a promoter and GPD transcription terminator of } X \text {. dendrorhous. }\end{array}$ & [56] \\
\hline pBS-gCYP51 & pBS bearing the genomic version of the CYP51 gene from $X$. dendrorhous (4,318 bp). & This work \\
\hline pBS-cCYP51 & pBS bearing the ORF of the CYP51 gene from X. dendrorhous (1,653 bp). & This work \\
\hline pBS-cyp51/hph & $\begin{array}{l}\text { pBS bearing the hygromycin B resistance cassette flanked by approximately } 700 \text { bp upstream } \\
\text { and } 600 \mathrm{pb} \text { downstream of the X. dendrorhous CYP51 locus. }\end{array}$ & This work \\
\hline YEp-Act4 & $\begin{array}{l}\text { pBR322 and } 2 \text { micron ori, } A m p^{R}, \text { LEU2 and containing the } A C T \text { promoter to regulate gene } \\
\text { expression. }\end{array}$ & [48] \\
\hline YEpNP & YEp-Act4 bearing the $S$. cerevisiae TDH3 terminator. & This work \\
\hline YEpNP-cCYP51 & $\begin{array}{l}\text { YEpNP bearing the } X \text {. dendrorhous CYP51 CDNA (1,653 bp) under the regulation of the ACT } \\
\text { promoter and the TDH3 terminator. }\end{array}$ & This work \\
\hline YEpNP-gERG11 & $\begin{array}{l}\text { YEpNP bearing the } S \text {. cerevisiae ERG11 gene }(1,593 \mathrm{bp}) \text { under the regulation of the ACT promoter } \\
\text { and the TDH3 terminator. }\end{array}$ & This work \\
\hline \multicolumn{3}{|l|}{ Strains: } \\
\hline \multicolumn{3}{|l|}{ E. coli: } \\
\hline $\mathrm{DH}-5 \mathrm{a}$ & $\begin{array}{l}\text { F- } \varphi 80 d \text { lacZ } \Delta \text { M15 (lacZY-argF) U169 deoR recA1 endA1 hsdR17(rk- mk+) phoA supE44 I- thi-1 } \\
\text { gyrA96 relA1 }\end{array}$ & [47] \\
\hline \multicolumn{3}{|l|}{ S. cerevisiae: } \\
\hline S288c & MATa, SUC2, gal2, mal, mel, flo1, flo8-1, hap1, ho, bio1, bio6. & [64] \\
\hline $\begin{array}{l}\text { Meyen ex E.C Hansen YHRO07C } \\
\left.\text { BY4743 (Sc-erg } 11^{+/-}\right)\end{array}$ & $\begin{array}{l}\text { MATa/MATa, his3 } \Delta 1 / \text { his } 3 \Delta 1 \text {, leu2 } \Delta 0 / \text { leu2 } \Delta 0 \text {, lys } 2 \Delta 0 /+ \text {, met15 } \Delta 0 /+ \text {, ura3 } \Delta 0 / \text { ura3 } \Delta 0 \text {, } \\
\Delta \text { ERG11:KanMx. ATCC } 26604 \text {. }\end{array}$ & ATCC 26604 \\
\hline Sc-cCYP51Xd & Diploid transformant derived from Sc-erg $11^{+/-}$containing the expression vector YEpNP-cCYP51. & This work \\
\hline Sc-gERG11Sc & Diploid transformant derived from Sc-erg $11^{+/-}$containing the expression vector YEpNP-gERG11. & This work \\
\hline Sc-hCYP51 & Sporulation product derived from Sc-erg $11^{+/-}$containing the expression vector YEpNP- cCYP51. & This work \\
\hline Sc-hERG11 & Sporulation product derived from Sc-erg $11^{+/-}$containing the expression vector YEpNP-gERG11. & This work \\
\hline \multicolumn{3}{|l|}{ X. dendrorhous: } \\
\hline UCD 67-385 & ATCC 24230, wild-type. & ATCC 24230 \\
\hline CBS 6938 & ATCC 96594, wild-type. & ATCC 96594 \\
\hline $\mathrm{CBS}-\mathrm{CYP} 51^{+/-}$ & $\begin{array}{l}\text { (CBS-CYP51/Cyp51 } 1^{\text {hph }} \text { ). Transformant derived from CBS } 6938 \text { containing an allele of the CYP51 } \\
\text { locus replaced by a hygromycin B resistance module. }\end{array}$ & This work \\
\hline
\end{tabular}


plates supplemented with $10 \mu \mathrm{g} / \mathrm{ml}$ hygromycin B (US Biological).

E. coli was cultured at $37^{\circ} \mathrm{C}$ with constant agitation in Luria-Bertani (LB, $1 \%$ tryptone, $0.5 \%$ yeast extract, and $0.5 \% \mathrm{NaCl}$ ) medium and on $1.5 \%$ agar LB plates supplemented with $100 \mu \mathrm{g} / \mathrm{ml}$ ampicillin for plasmid selection and $20 \mu \mathrm{g} / \mathrm{ml}$ of X-gal (5-bromo-4chloro-3-indolyl- $\beta$ - $D$ galactopyranoside) for recombinant clone selection by blue-white screening [47]. For selection, recombinant clones bearing the constructed plasmids were analyzed by direct colony PCR with an adequate set of primers.

S. cerevisiae strains were cultured at $30^{\circ} \mathrm{C}$ in $\mathrm{YM}$ or YEP (YM: $0.3 \%$ yeast extract, $0.3 \%$ malt extract and $0.5 \%$ peptone; YEP: $2 \%$ glucose, $1 \%$ yeast extract, and $2 \%$ peptone) rich media. The strain Meyen ex E.C Hansen YHR007C BY4743 was cultured at $22^{\circ} \mathrm{C}$ in SD medium ( $0.67 \%$ yeast nitrogen base without amino acids and $2 \%$ glucose) supplemented with metabolites to sustain the strain auxotrophy $(0.002 \%$ uracil, $1 \%$ histidine and $1 \%$ leucine) and $200 \mu \mathrm{g} / \mathrm{ml}$ of geneticin. Transformants derived from this strain were cultured in SD medium supplemented with uracil, histidine and geneticin, because the vector YEpNP complements the parental strain's leucine auxotrophy.

\section{Nucleic acid extraction, DNA amplification and sequence analysis}

$X$. dendrorhous DNA extraction was performed from protoplasts according to [49], and total RNA extraction was performed according to a modified protocol of Chomczynski and Sacchi [50] for $X$. dendrorhous [38]. Total RNA was quantified spectrophotometrically at $260 \mathrm{~nm}$ according to [47] in a V-630 UV-vis Spectrophotometer (JASCO). S. cerevisiae genomic DNA was obtained by mechanical cell disruption using $0.5 \mathrm{~mm}$ glass beads (BioSpec) and shaking in a mini bead beater-16 (BioSpec). Plasmid DNA was obtained from E. coli strains using the AxyPrep Plasmid Miniprep kit (Axygene).

Oligonucleotides designed and used in this work were synthesized by Integrated DNA Technologies and are listed in Additional file 2: Table S1. PCR reactions were performed in a final volume of $25 \mu \mathrm{l}$ with $2 \mathrm{U}$ of $\mathrm{Taq}$ DNA polymerase, $2.5 \mu \mathrm{l}$ of $10 \mathrm{X}$ Taq buffer, $0.5 \mu \mathrm{l}$ of $10 \mathrm{mM}$ dNTPs, $1 \mu \mathrm{l}$ of $50 \mathrm{mM} \mathrm{MgCl}, 1 \mu \mathrm{l}$ of each primer $(25 \mu \mathrm{M})$ and 10 to $20 \mathrm{ng}$ of template DNA in an Applied Biosystems 2720 thermal cycler. The general amplification protocol was as follows: initial denaturation at $95^{\circ} \mathrm{C}$ for $3 \mathrm{~min} ; 35$ cycles of denaturation at $94^{\circ} \mathrm{C}$ for $30 \mathrm{~s}$, annealing at $55^{\circ} \mathrm{C}$ for $30 \mathrm{~s}$, and synthesis at $72^{\circ} \mathrm{C}$ for $3 \mathrm{~min}$; and a final extension step at $72^{\circ} \mathrm{C}$ for $10 \mathrm{~min}$. Samples were stored at $4^{\circ} \mathrm{C}$ until analyzed by $0.8 \%$ agarose gel electrophoresis in TAE buffer stained with $0.5 \mu \mathrm{g} / \mathrm{ml}$ ethidium bromide [47]. DNA for sequencing or plasmid construction was purified from gels by the glass milk method [51].

DNA sequencing was performed in an ABI 3100 Avant genetic analyzer using the BigDye terminator v3.1 kit (Applied Biosystems) and analyzed with Vector NTI Suite 10 (Informax), CLUSTAL W 1.8 and programs available at the NCBI web site. Protein sequence analyses were performed with programs available at www.ch.embnet.org/software/TMPRED_form.html [52], www.ebi.ac.uk/InterProScan/ [53] and www.cyped.unistuttgart.de/ [25].

Protein modeling was performed using programs available at www.swissmodel.expasy.org/ [54], http:// autodock.scripps.edu/wiki/AutoDock4 [31] and www. ks.uiuc.edu/Research/vmd/ [55].

\section{Isolation of the $X$. dendrorhous CYP51 gene and plasmid construction}

To identify the CYP51 gene from $X$. dendrorhous, BLAST analyses using homologous CYP51 nucleotide and amino acid sequences were performed with the yeast genomic and transcriptomic databases available in our laboratory [20]. Specific primers were designed from the identified sequences (Additional file 2: Table S1).

For the $S$. cerevisiae heterologous complementation experiments, the CYP51 cDNA from $X$. dendrorhous and the ERG11 gene from $S$. cerevisiae (homologous to CYP51) were ligated into the yeast expression plasmid YEpNP (Table 3). First, plasmid pBS-cCyp51, which contained the cDNA of the CYP51 gene, was constructed by inserting a $1,653 \mathrm{bp}$ RT-PCR product into the EcoRV site of plasmid pBluescript SK-, which was obtained using the primer set cCYP51.F + cCYP51.R (Additional file 2: Table S1). Plasmid pBS-cyp51/Hyg was used to eliminate an allele of the CYP51 gene in $X$. dendrorhous. This plasmid was obtained by sequentially joining three DNA fragments: i) $700 \mathrm{bp}$ (amplified with primers CYP51_del.Fw + CYP51_del-HpaI.Rv) upstream of the CYP51 gene encoding sequence; ii) the hygromycin $B$ resistance module [56] (amplified with primers H.F and H.R); and iii) 600 bp (amplified with primers CYP51_delHpaI.Fw + CYP51_del.Rv) downstream of the CYP51 gene encoding sequence. These sequences were inserted into the EcoRV site of plasmid pBluescript SK-. For $X$. dendrorhous transformation, the insert of plasmid pBS-cyp51/Hyg was released using the NotI and XhoI endonucleases.

\section{Yeast transformation}

$S$. cerevisiae electrocompetent cells were prepared from $60 \mathrm{ml}$ of exponentially growing cultures in YEP medium at $22^{\circ} \mathrm{C}$ with constant agitation. The cells were washed three times with chilled sterile distilled water, one time with sorbitol $1 \mathrm{M}$ and finally suspended in $0.2 \mathrm{ml}$ of $1 \mathrm{M}$ sorbitol. The cells were kept at $4^{\circ} \mathrm{C}$, and $40 \mu \mathrm{l}$ aliquots 
were used for electroporation with a BioRad gene pulser $\times$ cell with $\mathrm{PC}$ and $\mathrm{CE}$ modules under the following conditions: $1.5 \mathrm{kV}, 25 \mu \mathrm{F}$ and $200 \Omega$. X. dendrorhous transformation was also performed by electroporation according to [57] and [58] using 1 to $5 \mu \mathrm{g}$ of linear donor DNA. Electrocompetent cells were prepared from exponential cultures, grown in YM medium and electroporated using the following program: $125 \mathrm{mF}, 600 \Omega, 0.45 \mathrm{kV}$.

\section{S. cerevisiae heterologous complementation assay}

The $\operatorname{erg} 11^{(+-)}$S. cerevisiae strain, which is diploid and heterozygous for the ERG11 gene, was used for the gene complementation assays. The $S$. cerevisiae ERG11 gene and the $X$. dendrorhous CYP51 gene were ligated into plasmid YEpNP and used to independently transform the original diploid yeast strain. Transformants were selected in SD agar plates supplemented with $200 \mu \mathrm{g} / \mathrm{ml}$ geneticin, $0.002 \%$ uracil and $1 \%$ histidine according to the parental strain requirements. Transformant strains were randomly chosen and incubated in pre-sporulation medium plates $(0.8 \%$ yeast extract, $0.3 \%$ peptone, $10 \%$ glucose and $2 \%$ agar), incubated at $22^{\circ} \mathrm{C}$ for 1 to 2 days and then transferred to minimal sporulation medium plates ( $1 \%$ potassium acetate and $2 \%$ agar) for incubation at $22^{\circ} \mathrm{C}$ for 3 to 6 days. Supplements according to the parental strain genotype were added to the sporulation media. Asci formation was confirmed by optical microscopy. To enrich the asci fraction, cells were collected from the sporulation plates, treated with diethyl ether for $20 \mathrm{~min}$ at $22^{\circ} \mathrm{C} \mathrm{[34]} \mathrm{and} \mathrm{the} \mathrm{ascospores} \mathrm{were} \mathrm{released}$ by treatment with zimoliase- $120 \mathrm{~T}$ (US Biological) at $30^{\circ}$ $\mathrm{C}$ for $2 \mathrm{~h}$. Afterwards, the mixture was shaken in a mini bead beater-16 (BioSpec) with $0.5 \mathrm{~mm}$ glass beads (BioSpec) for 2 min [59]. Finally, the cells were spread on SD agar plates supplemented with histidine, uracil, lysine, methionine and geneticin to support all possible combinations of auxotrophies in the resulting haploid cells.

To select the desired haploid cells to study gene complementation, the obtained colonies were streaked in replicates on SD agar plates supplemented with geneticin and: i) uracil, histidine and lysine, ii) uracil, histidine and methionine and iii) uracil, histidine, methionine and lysine. Colonies that developed on the plates supplemented with the four metabolites but not when lysine or methionine were omitted were chosen for further analyses.

\section{Sterol and carotenoid extraction and analysis}

Sterols were extracted from both the $S$. cerevisiae and $X$. dendrorhous strains, while carotenoids were extracted only from $X$. dendrorhous. Metabolites were spectrophotometrically quantified and normalized to the dry weight of the yeast.

Sterols were extracted according to the protocol of [60] by mixing cell pellets with $4 \mathrm{~g}$ of $\mathrm{KOH}$ and $16 \mathrm{ml}$ of
$60 \%(\mathrm{v} / \mathrm{v})$ ethanol/water and incubating at $80 \pm 2^{\circ} \mathrm{C}$ for $2 \mathrm{~h}$. Then, non-saponificable sterols were extracted with $10 \mathrm{ml}$ of petroleum ether and quantified spectrophotometrically at $280 \mathrm{~nm}$. Carotenoids were extracted from cellular pellets by the acetone method [61] and quantified spectrophotometrically at $465 \mathrm{~nm}$. Sterol and carotenoid compositions were analyzed by RP-HPLC with an RP-18 Lichrocart125-4 (Merck) column using methanol: water $(97: 3, \mathrm{v} / \mathrm{v})$ or acetonitrile: methanol: isopropanol (85: $10: 5, \mathrm{v} / \mathrm{v}$ ) as the mobile phase with a $1 \mathrm{ml} / \mathrm{min}$ flux under isocratic conditions to separate the sterols and carotenoids, respectively. The elution spectra were recovered using a diode array detector, and the metabolites were identified according to their spectra and retention time in comparison to standards.

\section{Single-strand DNA synthesis and RT-qPCR}

Single-stranded DNA was synthesized according to the M-MLV reverse transcriptase (Invitrogen) manufacturer's instructions using $5 \mu \mathrm{g}$ of total RNA in a final volume of $20 \mu \mathrm{l}$. Relative gene expression levels were obtained in an Mx3000P quantitative PCR system (Stratagene) using $1 \mu \mathrm{l}$ of the reverse transcription reaction, $0.25 \mu \mathrm{M}$ of each primer and $10 \mu \mathrm{l}$ of the SensiMix SYBR Green I (Quantace) kit in a final volume of $20 \mu \mathrm{l}$. The $\mathrm{Ct}$ values obtained were normalized to the corresponding value for the beta-actin encoding gene [Genbank: X89898.1] [62], and later expressed as a function of the control conditions using the $\Delta \Delta \mathrm{Ct}$ algorithm [63].

\section{Additional files}

Additional file 1: Figure S1. Growth curves of yeast strains used in this study. (A) S. cerevisiae strains Sc-erg $11^{+/-}$, Sc-hERG11 and Sc-hCYP51 were cultured in YM media at $22^{\circ} \mathrm{C}$ with constant agitation. (B) Growth curve of the X. dendrorhous CBS 6938 and CBS-CYP51 $1^{+-}$strains cultured in YM media at $22^{\circ} \mathrm{C}$ with constant agitation. Values are the mean \pm standard error of three independent cultures.

Additional file 2: Table S1. Primers designed and used in this study.

\section{Competing interests}

The authors declare no competing financial or non-financial interests.

\section{Authors' contributions}

$\mathrm{KL}$ bioinformatically characterized the CYP51 gene, performed the CYP51 protein modeling and S. cerevisiae heterologous complementation analysis. NW contributed to the S. cerevisiae complementation analyses. DS and SB performed the $X$. dendrorhous transformations, plasmid construction and transformant analyses: carotenoid, ergosterol and gene expression analyses. $\mathrm{MB}$ and VC contributed to the study design and analysis of the results. JA conceived of the study and participated in the experimental design and coordination. JA, KL, MB and VC drafted the manuscript. All authors read and approved the final manuscript.

\section{Acknowledgements}

This work was financially supported by Fondecyt 11121200. NW was supported by a CONICYT fellowship.

Received: 5 February 2015 Accepted: 17 April 2015

Published online: 25 April 2015 


\section{References}

1. The Global Market for Carotenoids. Report Code: FOD025D. [http:// www.bccresearch.com/pressroom/fod/global-carotenoids-marketreach-11th4-billion-2018]

2. Schmidt I, Schewe H, Gassel S, Jin C, Buckingham J, Hümbelin M, et al. Biotechnological production of astaxanthin with Phaffia rhodozymal Xanthophyllomyces dendrorhous. Appl Microbiol Biotechnol. 2011;89:555-71.

3. Schroeder WA, Johnson EA. Antioxidant role of carotenoids in Phaffia rhodozyma. J Gen Microbiol. 1993;139:907-12.

4. Schroeder WA, Johnson EA. Carotenoids protect Phaffia rhodozyma against singlet oxygen damage. J Ind Microbiol. 1995;14:502-7.

5. Schroeder WA, Johnson EA. Singlet oxygen and peroxyl radicals regulate carotenoid biosynthesis in Phaffia rhodozyma. J Biol Chem. 1995;270:18374-9.

6. Wang X, Willen R, Wadstrom T. Astaxanthin-rich algal meal and vitamin C inhibit Helicobacterpylori infection in BALB/CA mice. Antimicrob Agents Chemother. 2000;44:2452-7.

7. Higuera-Ciapara I, Felix-Valenzuela L, Goycoolea FM. Astaxanthin: a review of its chemistry and applications. CRC Crit Rev Food Sci Nutr. 2006;46:185-96.

8. Park JS, Chyun JH, Kim YK, Line LL, Chew BP. Astaxanthin decreased oxidative stress and inflammation and enhanced immune response in humans. Nutr Metab. 2010;7:18

9. Yasui Y, Hosokawa M, Mikami N, Miyashita K, Tanaka T. Dietary astaxanthin inhibits colitis and colitis-associated colon carcinogenesis in mice via modulation of the inflammatory cytokines. Chem Biol Interact. 2011;193:79-87.

10. Alvarez V, Rodriguez-Saiz M, de la Fuente JL, Gudina EJ, Godio RP, Martin JF, et al. The crtS gene of Xanthophyllomyces dendrorhous encodes a novel cytochrome-P450 hydroxylase involved in the conversion of beta-carotene into astaxanthin and other xanthophylls. Fungal Genet Biol. 2006;43:261-72.

11. Ojima K, Breitenbach J, Visser H, Setoguchi Y, Tabata K, Hoshino T, et al. Cloning of the astaxanthin synthase gene from Xanthophyllomyces dendrorhous (Phaffia rhodozyma) and its assignment as a beta-carotene 3-hydroxylase/4-ketolase. Mol Genet Genomics. 2006;275:148-58.

12. Alcaino J, Barahona S, Carmona M, Lozano C, Marcoleta A, Niklitschek M, et al. Cloning of the cytochrome p450 reductase (crtR) gene and its involvement in the astaxanthin biosynthesis of Xanthophyllomyces dendrorhous. BMC Microbiol. 2008;8:169.

13. Csernetics A, Toth E, Farkas A, Nagy G, Bencsik O, Vagvolgyi C, et al. Expression of Xanthophyllomyces dendrorhous cytochrome-P450 hydroxylase and reductase in Muco rcircinelloides. World J Microbiol Biotechnol. 2015;31:321-36.

14. Zhang H, Im S-C, Waskell L. Cytochrome b5 increases the rate of product formation by cytochrome P450 2B4 and competes with cytochrome P450 reductase for a binding site on cytochrome P450 2B4. J Biol Chem. 2007;282:29766-76.

15. Degtyarenko KN, Archakov Al. Molecular evolution of P450 superfamily and P450-containing monooxygenase systems. FEBS Lett. 1993;332:1-8.

16. Bernhardt R. Cytochromes P450 as versatile biocatalysts. J Biotechnol. 2006;124:128-45.

17. Estabrook RW. A passion for P450s (remembrances of the early history of research on cytochrome P450). Drug Metab Dispos. 2003;31:1461-73.

18. Porter TD, Coon MJ. Cytochrome P-450. Multiplicity of isoforms, substrates, and catalytic and regulatory mechanisms. J Biol Chem. 1991;266:13469-72.

19. Kimmich N, Das A, Sevrioukova I, Meharenna Y, Sligar SG, Poulos TL. Electron transfer between cytochrome P450cin and its FMN-containing redox partner, cindoxin. J Biol Chem. 2007;282:27006-11.

20. Loto I, Gutiérrez MS, Barahona S, Sepúlveda D, Martínez-Moya P, Baeza M, et al. Enhancement of carotenoid production by disrupting the C22-sterol desaturase gene (CYP61) in Xanthophyllomyces dendrorhous. BMC Microbiol. 2012;12:235.

21. Kelly SL, Kelly DE. Microbial cytochromes P450: biodiversity and biotechnology. Where do cytochromes P450 come from, what do they do and what can they do for us? Philos Trans R Soc Lond B Biol Sci. 2013;368:20120476.

22. Aoyama Y, Yoshida Y, Sato R. Yeast cytochrome P-450 catalyzing lanosterol 14 alpha-demethylation. II. Lanosterol metabolism by purified P-450(14)DM and by intact microsomes. J Biol Chem. 1984;259:1661-6.

23. Kalb VF, Woods CW, Turi TG, Dey CR, Sutter TR, Loper JC. Primary structure of the P450 lanosterol demethylase gene from Saccharomyces cerevisiae. DNA. 1987;6:529-37.

24. Werck-Reichhart D, Feyereisen R. Cytochromes P450: a success story. Genome Biol. 2000;1:3003.1-9.
25. Fischer $M$, Knoll M, Sirim D, Wagner F, Funke S, Pleiss J. The Cytochrome P450 Engineering Database: a navigation and prediction tool for the cytochrome P450 protein family. Bioinformatics. 2007;23:2015-7.

26. Sirim D, Wagner F, Lisitsa A, Pleiss J. The cytochrome P450 engineering database: Integration of biochemical properties. BMC Biochem. 2009;10:27.

27. Shimizu T, Tateishi T, Hatano M, Fujii-Kuriyama Y. Probing the role of lysines and arginines in the catalytic function of cytochrome P450d by site-directed mutagenesis. Interaction with NADPH-cytochrome P450 reductase. J Biol Chem. 1991;266:3372-5.

28. Hatae T, Hara S, Yokoyama C, Yabuki T, Inoue H, Ullrich V, et al. Site-directed mutagenesis of human prostacyclin synthase: Alteration of Cys441 of the Cys-pocket, and Glu347 and Arg350 of the EXXR motif. FEBS Lett. 1996:389:268-72

29. Syed K, Mashele SS. Comparative analysis of P450 signature motifs EXXR and $C X G$ in the large and diverse kingdom of fungi: identification of evolutionarily conserved amino acid patterns characteristic of P450 family. PLoS One. 2014;9:e95616.

30. van den Brink HM, van Gorcom RF, van den Hondel CA, Punt PJ. Cytochrome P450 enzyme systems in fungi. Fungal Genet Biol. 1998;23:1-17.

31. Morris GM, Huey R, Lindstrom W, Sanner MF, Belew RK, Goodsell DS, et al. AutoDock4 and AutoDockTools4: Automated docking with selective receptor flexibility. J Comput Chem. 2009;30:2785-91.

32. Sharma $V$, Bhatia R. Triazoles in Antifungal Therapy: A Review. Int J Res Pharm Biomed. 2011;2:417-27.

33. Winzeler EA, Shoemaker DD, Astromoff A, Liang H, Anderson K, Andre B, et al. Functional characterization of the $S$. cerevisiae genome by gene deletion and parallel analysis. Science. 1999;285:901-6.

34. Dawes IW, Hardie ID. Selective killing of vegetative cells in sporulated yeast cultures by exposure to diethyl ether. Mol Gen Genet. 1974:131:281-9.

35. Alcaino J, Romero I, Niklitschek M, Sepulveda D, Rojas MC, Baeza M, et al. Functional characterization of the Xanthophyllomyces dendrorhous farnesyl pyrophosphate synthase and geranylgeranyl pyrophosphate synthase encoding genes that are involved in the synthesis of isoprenoid precursors. PLoS One. 2014;9:e96626.

36. Lepesheva Gl, Waterman MR. Sterol 14alpha-demethylase cytochrome P450 (CYP51), a P450 in all biological kingdoms. Biochim Biophys Acta. 2007;1770:467-77.

37. Zill G, Engelhardt G, Wallnöfer PR. Determination of ergosterol as a measure of fungal growth using Si 60 HPLC. Z Lebensm Unters For. 1988;187:246-9.

38. Lodato P, Alcaino J, Barahona S, Retamales P, Jimenez A, Cifuentes V. Study of the expression of carotenoid biosynthesis genes in wild-type and deregulated strains of Xanthophyllomyces dendrorhous (Ex.: Phaffia rhodozyma). Biol Res. 2004;37:83-93.

39. Wozniak A, Lozano C, Barahona S, Niklitschek M, Marcoleta A, Alcaíno J, et al. Differential carotenoid production and gene expression in Xanthophyllomyces dendrorhous grown in a nonfermentable carbon source. FEMS Yeast Res. 2011;11:252-62.

40. Marcoleta A, Niklitschek M, Wozniak A, Lozano C, Alcaino J, Baeza M, et al. Glucose and ethanol-dependent transcriptional regulation of the astaxanthin biosynthesis pathway in Xanthophyllomyces dendrorhous. BMC Microbiol. 2011:11:190.

41. Verdoes JC, Sandmann G, Visser H, Diaz M, van Mossel M, van Ooyen AJ. Metabolic engineering of the carotenoid biosynthetic pathway in the yeast Xanthophyllomyces dendrorhous (Phaffia rhodozyma). Appl Environ Microbiol. 2003;69:3728-38.

42. Miao L, Chi S, Tang Y, Su Z, Yin T, Guan G, et al. Astaxanthin biosynthesis is enhanced by high carotenogenic gene expression and decrease of fatty acids and ergosterol in a Phaffia rhodozyma mutant strain. FEMS Yeast Res. 2011;11:192-201

43. Calo P, de Miguel T, Velázquez JB, Villa TG. Mevalonic acid increases trans-astaxanthin and carotenoid biosynthesis in Phaffia rhodozyma. Biotechnol Lett. 1995;17:575-8.

44. Shimada H, Kondo K, Fraser PD, Miura Y, Saito T, Misawa N. Increased carotenoid production by the food yeast Candida utilis through metabolic engineering of the isoprenoid pathway. Appl Environ Microbiol. 1998:64:2676-80

45. Parks LW, Casey WM. Physiological implications of sterol biosynthesis in yeast. Annu Rev Microbiol. 1995;49:95-116.

46. Tang Q, Li Y, Yuan QP. Effects of an ergosterol synthesis inhibitor on gene transcription of terpenoid biosynthesis in Blakeslea trispora. Curr Microbiol. 2008;57:527-31. 
47. Sambrook J, Russell DW. Molecular Cloning a laboratory manual. New York, United States: Cold Spring Harbor; 2001.

48. Sanchez-Torres P, Gonzalez-Candelas L, Ramon D. Heterologous Expression of a Candida molischiana Anthocyanin-beta-glucosidase in a Wine Yeast Strain. J Agric Food Chem. 1998;46:354-60.

49. Cifuentes V, Hermosilla G, Martinez C, Leon R, Pincheira G, Jimenez A. Genetics and electrophoretic karyotyping of wild-type and astaxanthin mutant strains of Phaffia rhodozyma. Antonie Van Leeuwenhoek. 1997;72:111-7.

50. Chomczynski P, Sacchi N. Single-step method of RNA isolation by acid guanidinium thiocyanate-phenol-chloroform extraction. Anal Biochem. 1987;162:156-9.

51. Boyle JS, Lew AM. An inexpensive alternative to glassmilk for DNA purification. Trends Genet. 1995;11:8.

52. Hofmann K, Stoffel W. TMbase-A database of membrane spanning protein segments. Biol Chem H-S. 1993;374:166.

53. Zdobnov EM, Apweiler R. InterProScan-an integration platform for the signature-recognition methods in InterPro. Bioinformatics. 2001;17:847-8.

54. Biasini M, Bienert S, Waterhouse A, Arnold K, Studer G, Schmidt T, et al. SWISS-MODEL: modelling protein tertiary and quaternary structure using evolutionary information. Nucleic Acids Res. 2014;42:W252-8.

55. Humphrey W, Dalke A, Schulten K. VMD: Visual Molecular Dynamics. J Mol Graph. 1996;14:33-8.

56. Niklitschek M, Alcaíno J, Barahona S, Sepúlveda D, Lozano C, Carmona M, et al. Genomic organization of the structural genes controlling the astaxanthin biosynthesis pathway of Xanthophyllomyces dendrorhous. Biol Res. 2008;41:93-108.

57. Adrio JL, Veiga M. Transformation of the astaxanthin-producing yeast Phaffia rhodozyma. Biotechnol Tech. 1995;9:509-12.

58. Kim IG, Nam SK, Sohn JH, Rhee SK, An GH, Lee SH, et al. Cloning of the ribosomal protein $\mathrm{L} 41$ gene of Phaffia rhodozyma and its use as a drug resistance marker for transformation. Appl Environ Microbiol. 1998;64:1947-9.

59. Sherman F. Getting started with yeast. Methods Enzymol. 2002;350:3-41.

60. Shang F, Wen $S$, Wang $X$, Tan T. Effect of nitrogen limitation on the ergosterol production by fed-batch culture of Saccharomyces cerevisiae. J Biotechnol. 2006;122:285-92.

61. An GH, Schuman DB, Johnson EA. Isolation of Phaffia rhodozyma mutants with increased astaxanthin content. Appl Environ Microbiol. 1989;55:116-24.

62. Lodato P, Alcaíno J, Barahona S, Niklitschek M, Carmona M, Wozniak A, et al. Expression of the carotenoid biosynthesis genes in Xanthophyllomyces dendrorhous. Biol Res. 2007:40:73.

63. Livak KJ, Schmittgen TD. Analysis of relative gene expression data using real-time quantitative PCR and the 2(-Delta Delta C(T)) Method. Methods. 2001;25:402-8

64. Mortimer RK, Johnston JR. Genealogy of principal strains of the yeast genetic stock center. Genetics. 1986;113:35-43.

\section{Submit your next manuscript to BioMed Central and take full advantage of:}

- Convenient online submission

- Thorough peer review

- No space constraints or color figure charges

- Immediate publication on acceptance

- Inclusion in PubMed, CAS, Scopus and Google Scholar

- Research which is freely available for redistribution 